\title{
Impact of Preoperative Chemo-radiation Therapy on Systemic Failure in Locally Advanced Rectal Cancer
}

Yoon Dae Han, Mahdi Hussain Al Bandar, Min Soo Cho, Hyuk Hur, Byung Soh Min, Kang Young Lee and Nam Kyu Kim*

Department of Surgery, College of Medicine, Yonsei University, Seoul, South Korea

*Corresponding author: Nam Kyu Kim, Department of Surgery, Division of Colorectal Surgery, Colorectal Cancer Special Clinic, Yonsei University College of Medicine, Seoul, Korea, Tel: +82-2-2228-2117; Fax: +82-2-313-8289; E-mail: namkyuk@yuhs.ac

Received date: Aug 19, 2016; Accepted date: Oct 10, 2016; Published date: Oct 20, 2016

Copyright: (C) 2016 Han YD et al. This is an open-access article distributed under the terms of the Creative Commons Attribution License, which permits unrestricted use, distribution, and reproduction in any medium, provided the original author and source are credited.

\begin{abstract}
Background and objectives: Advanced rectal cancers require local and systemic control. Chemo radiotherapy (CRT) is adequate to achieve adequate local control. Systemic control, however, is a dominant obstacle remained in debates. We compared oncology outcome in both arms in patients with advanced rectal cancers in order to identify high-risk group of distant metastasis.
\end{abstract}

Methods: Data for 723 patients for advanced rectal cancer from 2005 to 2013 retrieved retrospectively. Patients were classified to CRT $(n=364)$ or no CRT $(n=359)$ arms.

Results: CRT group showed greater local control and achieved pT stage 0,1 , or 2 in $43.7 \%$ vs. $28.4 \%$ in no CRT $(p<0.001)$ and less CEA marker $(11.17 \pm 25.2$ vs. $6.14 \pm 11.3, p<0.001)$, respectively. Although CRT group had higher rates of advanced tumors, cT3 or T4 $(341(93.7 \%)$ vs. $294(81.9 \%), p<0.001)$ and CRM threat $(167(45.9 \%)$ vs. $30(8.4 \%) ; p<0.001)$. Overall local recurrence rate observed in no CRT $3 \%$ compared to $2.1 \%$ in CRT arm, ( $p<0.005)$. Systemic recurrence rate was similar in both groups, $(22.5 \%$ vs. $23 \%)$, respectively.

Conclusion: CRT is efficient to downstage locally advanced rectal cancer, not systemic control though. Early recognition of high-risk group is recommended in order to consider CRT modification ahead of planned surgery.

Keywords: Chemo-radiotherapy; Systemic failure; Locally advanced rectal cancer

\section{Introduction}

Colorectal cancer (CRC) is a leading cause of cancer-related mortality worldwide, with over 1.2 million new cases diagnosed each year [1]. Surgical approaches for rectal cancer have evolved markedly with the development of a promising standardized approach for total mesorectal excision (TME), which was first described by Professor Bill Heald [2]. In addition, screening programs and increased colorectal cancer (CRC) literacy have contributed to improved detection of early cancer, with 5-year survival ranging from $50 \%$ to $90 \%$ with adequate surgery [3]. The mainstay of early CRC treatment is curative surgery; however, treatment of advanced CRC is challenging, particularly for advanced rectal cancer, because of the limitations of the operation field and location of the tumor in close association with vital structures responsible for urinary and sexual function. As a result, several techniques and procedures have been developed to enhance surgical management and reduce comorbidity. Neo adjuvant chemo radiotherapy (CRT) has shown effectiveness in advanced rectal cancer in term of tumor down staging, sphincter preservation, and reduction of local recurrence, but has failed to achieve systemic control $[4,5]$.

Before the advent of CRT and TME, both local and distant recurrences represented major problems in the treatment of rectal cancer, with rates of local recurrence reaching 32\%-35\% for conventional surgery [6]. Standardized treatment of advanced rectal cancer has been proposed based on findings of the German
CAO/ARO/AIO-94 randomized phase III trial after a median followup of 11 years and according to the International Union Against Cancer (IUCC) [4,7]. The German trial compared efficacy of preoperative CRT and postoperative CRT in 823 patients with stage 2-3 rectal cancer and showed a cumulative incidence of local relapse of $7.1 \%$ and $10.1 \%$ in the pre- and postoperative arms, respectively $(p=0.048)$. The rationale for this approach is based on more efficient destruction of normally oxygenated tumor cells compared to those with impaired perfusion after pelvic surgery; thus, it is hypothesized that this method will eradicate micrometastasis, shrink tumor burden, and downstage the tumor [8]. Nevertheless, systemic failure represents another challenging entity that has provoked long-standing and ongoing debates. In most of the published articles to date, CRT by itself could not influence or control distal tumor spread [9]. This outcome was studied previously by a German group [4], detected no significant differences in the 10-year cumulative incidence of distant metastases and disease-free survival ( $29.8 \%$ and $29.6 \%$, respectively; $\mathrm{p}=0.9)$.

Numerous trials have considered modifying the treatment regimen by adding a chemotherapy course to radiotherapy (RT) before surgery or as consolidation chemotherapy, and concomitant use of chemotherapy with RT has been proposed worldwide. Addition of perioperative administration of chemotherapy to the treatment strategy for advanced rectal cancer has shown promise in the recently published results of the CORONA I study, with improvement in overall survival [10-14]. However, the risk of chemotherapy-related toxicity and delay in the planned surgical management, as well as failure to control distant metastasis, contribute to difficulties in decision making. A study by a Dutch colorectal cancer group [15] reported that the rate 
of fecal incontinence increased from $27 \%$ and $39 \%$ after surgery alone to $62 \%$ and $64 \%$ after preoperative radiotherapy. This is a serious example of decreased patient quality of life over the time. We therefore need to weigh the beneficial impact of CRT against the risk of complications. These issues are under extensive investigation. We designed the current study to evaluate our single institute experience in treating advanced rectal cancer with the standard protocol of CRT followed by TME versus surgery alone and to study the treatmentrelated influence on overall survival and local and systemic recurrence.

\section{Materials and Methods}

\section{Patients}

Patient records from 2005 to 2013 were reviewed, and 723 patients who were diagnosed with advanced mid and low rectal cancer in a single institute were enrolled for analysis. Patient charts, treatment plans, and results were entered prospectively into the electronic data base system. Patients were deemed to have locally advanced disease (T3-T4 or N-positive) on the basis of magnetic resonance imaging (MRI). This study was carried out with permission from institutional review board and department of general surgery in Yonsei Medical Health Care System. All patients were considered eligible on the basis of histopathologically confirmed rectal adenocarcinoma with inferior margin within $10 \mathrm{~cm}$ of the anal verge. Data were retrieved retrospectively, and patients were allocated into a CRT group $(n=364)$ or no CRT group (surgery alone, $n=359$ ). The primary goal was to compare the outcomes of the CRT group and no CRT group in terms of local and systemic recurrence and overall survival (OS) rate.

We excluded patients with recurrent rectal cancer, previous radiation therapy to the pelvic region, or previous diagnosis of other malignancies or distant metastasis. All patients were required to undergo a computed tomography (CT) scan of the chest, abdomen, and pelvis and a serum carcinoembryonic antigen (CEA) measurement. For local tumor staging, the magnetic resonance imaging (MRI) has used for all patient group. However Endoscopic ultrasound was used occasionally to assess clinical complete response. The American Joint Committee on Cancer (7th edition) TNM system was used for staging [16]. Tumor location was defined as upper rectum if $>10 \mathrm{~cm}$ from the anal verge $(\mathrm{AV})$, mid rectum if $6-10 \mathrm{~cm}$ from $\mathrm{AV}$, and low rectum if $<6 \mathrm{~cm}$ from $\mathrm{AV}$.

\section{Treatment protocol}

All patients had undergone long-course CRT before surgery for poorly prognostic features suspected by MRI or other parameters (High risk group); however, surgery was performed upfront if no circumferential resection margin (CRM) threat was identified (Low risk group). If preoperative CRT was administered, surgery was performed after a recovery time of 6-8 weeks and was followed by adjuvant chemotherapy (AC) 6 weeks after surgery in most cases.

Long-course CRT is based on radiotherapy at $45 \mathrm{~Gy}$ delivered over a period of 5 weeks and one course of chemotherapy consisting of 350 $\mathrm{mg}$ of fluorouracil per square meter of body surface area per day and $20 \mathrm{mg}$ of leucovorin per square meter per day, both administered for 5day courses during the first and fifth weeks of radiotherapy. Patients were discharged on the 7th-10th days after surgery if the postoperative course was uneventful.

\section{Surgery}

Curative surgery was performed by highly expert surgeons in the field of CRC with a minimum experience of 200 procedures. Procedures were standardized within our unit of the colorectal department. Laparoscopic and robotic rectal surgeries have been performed since 2007 at our institution.

The medial approach with inferior mesenteric artery (IMA) dissection was used according to surgeon preference; however, high ligation of the IMA was encouraged if an enlarged lymph node was anticipated at the root. The type of procedure was planned based on pre-CRT, post-treatment imaging scans, and patient counseling to maximize the negative resection margin rate and achieve $\mathrm{R} 0$ resection.

\section{Follow-up and monitoring}

All patients were followed-up within 2 weeks postoperatively to address wound complications and patient status, as well as to discuss the final histopathology report. Follow-up was every 3 months for the first 2 years, every 6 months for the following 3 years, and annually thereafter.

Baseline assessment at each visit including clinical examination, rectal exam, proctoscopy, and CEA marker evaluation, as well as chest and abdomino-pelvic computed tomography (CT) and pelvic MRI in the case of suspected tumor recurrence or presence of distant metastasis. Patients were determined to have local recurrence if any tumor deposit was shown in radiological images or proven by biopsy for a intraluminal location 6 months or more after primary surgery.

\section{Study definition and protocol}

Overall survival was defined as the time from diagnosis to the recorded date of death. Threatened CRM was defined as involvement of $<2 \mathrm{~mm}$ of margin determined clinically by MRI or $<1 \mathrm{~mm}$ determined histologically. Lymph node positivity was assigned based on the TNM staging system (N1, 1-4 positive lymph node; N2>4 positive lymph nodes). Tumor locations were classified as low $(0-5 \mathrm{~cm}$ from anal verge) or mid $(5-10 \mathrm{~cm})$-rectal based on preoperative rigid sigmoidoscopy and MRI images.

Local recurrence was defined as radiologic evidence of disease recurrence in the pelvic cavity after previous curative surgery and was validated pathologically. The duration to local or systemic recurrence was the interval from the date of surgery to the first date of diagnosis of recurrence (radiologic or pathologic).

\section{Statistical Analysis}

Data are summarized as frequency and percentage for categorical variables and as median and range for continuous variables. Chisquared tests were used to compare proportions, and independent $t$ test was performed for comparison of continuous variables. Analyses of overall survival (OS) and disease-free survival (DFS) were carried out by the Kaplan-Meier method. All results were considered statistically significant if the $\mathrm{p}$ value was less than 0.05 . Statistical Package for the Social Sciences for Windows (Version 20.0, Chicago, IL) was used for data analysis. 
Citation: Han YD, Al Bandar MH, Cho MS, Hur H, Min BS, et al. (2016) Impact of Preoperative Chemo-radiation Therapy on Systemic Failure in Locally Advanced Rectal Cancer. J Integr Oncol 5: 180. doi:10.4172/2329-6771.1000180

Page 3 of 8

\section{Results}

\section{Patient characteristics}

From January 2005 to 2013,723 patients were enrolled in the study. Of these, 359 patients were assigned to the no CRT group, and 364 patients underwent CRT. The majority of our patients were male: 221 (61.6\%) in the no CRT group vs. $241(66.2 \%)$ in the CRT group. Median follow up period was for 46 months. Median age was 61 years (range, 22-89) in the no CRT group compared with 59 (27-88) years in the CRT group. Preoperative level of the CEA marker was elevated in the CRT group $(11.17 \pm 25.2$ vs. $6.14 \pm 11.3, \mathrm{p}<0.001)$; however, the postoperative CEA level was reduced to $1.44 \pm 1.9$ in the CRT group. High-risk features were more commonly found in the CRT group; lower rectum tumor was present in 150 patients $(41.2 \%)$ vs. $66(18.4 \%)$ vs. in CRT and no CRT group, respectively $(\mathrm{p}<0.001)$, whereas midrectum tumor was more frequent in the no CRT group (293 patients, $81.6 \%$ ) compared with the CRT group (214 patients, $58.8 \%$; $\mathrm{p}<0.001$ ). Tumor was staged clinically by MRI and found high risk feature in CRT group, invading through the bowel wall at cT3-4 stage in the majority of cases in both groups, but more frequently in the CRT group (341 patients, 93.7\%) than in the no CRT group (294 patients, $81.9 \% ; \mathrm{p}<0.001)$.

\begin{tabular}{|l|l|l|l|}
\hline Variables & No CRT (n=359) & CRT (n=364) & p value \\
\hline Age & $61(22-89)$ & $59(27-88)$ & 0.021 \\
\hline Pre-op CEA & $6.14 \pm 11.3$ & $11.17 \pm 25.2$ & 0.001 \\
\hline Post-op CEA & $1.87 \pm 2.7$ & $1.44 \pm 1.9$ & 0.023 \\
\hline Sex & & & 0.193 \\
\hline Male & $221(61.6 \%)$ & $241(66.2 \%)$ & \\
\hline Female & $138(38.4 \%)$ & $123(33.8 \%)$ & \\
\hline cT stage & & & $<0.001$ \\
\hline I, II & $65(18.1 \%)$ & $23(6.3 \%)$ & $<0.001$ \\
\hline III, IV & $294(81.9 \%)$ & $341(93.7 \%)$ & \\
\hline Tumor location & & $167(45.9 \%)$ & \\
\hline Low (<6 cm) & $66(18.4 \%)$ & $214(58.8 \%)$ & \\
\hline Mid (6-10 cm) & $293(81.6 \%)$ & $33(8.5 \%)$ & \\
\hline MRI CRM invasion & $359(100 \%)$ & & \\
\hline No & $0(0 \%)$ & & \\
\hline Suspected & $356(99.2 \%)$ & & \\
\hline EMVI status & & & \\
\hline No & & & \\
\hline Suspected & & & \\
\hline & & & \\
\hline
\end{tabular}

CT stage: Clinical T Stage, pre-op CEA: Preoperative Carcino Embryonic Antigen, post-op CEA: Postoperative Carcino Embryonic Antigen, CRM: Circumferential Resection Margin, EMVI: Extramural Venous Invasion.

Table 1: Patient characteristics.
Threatened CRM was seen in the CRT group only (167 patients, $45.9 \%$ vs. 0 patients in the no CRT group; $\mathrm{p}<0.001)$. The extramural venous invasion (EMVI) rate was higher in the CRT group $(31(8.5 \%)$ vs. $3(0.8 \%), \mathrm{p}<0.001$, respectively). CRT resulted in down staging of the tumor locally to reach achieve R0 resection in all patients with reasonable CRM positive rate at $10(2.7 \%)$. Down staging effect described in Tables 1 and 2.

\section{Histologic findings}

The majority of the tumors were moderately differentiated in both no CRT and CRT groups (304 patients (84.7\%) vs. 299 patients (82.1\%). We observed a significant prominent effect of CRT regimen on tumor down staging for most of the variables. Down staging evident in Lympho-vascular invasion (LVI) was present in 106 patients (31.9\%) in the no CRT group compared with only 38 patients $(11.6 \%)$ in the CRT group $(\mathrm{p}<0.001)$. The number of positive lymph nodes retrieved from the no CRT group was higher than that for the CRT group (3.96 \pm 2.4 vs. $2.78 \pm 2.4$, respectively; $\mathrm{p}<0.001$ ).

\begin{tabular}{|l|l|l|l|}
\hline Histology & No CRT (n=359) & CRT (n=364) & p value \\
\hline Total LN & $22.73 \pm 13.2$ & $15.45 \pm 8.6$ & $<0.001$ \\
\hline Positive LN & $3.96 \pm 2.4$ & $2.78 \pm 2.4$ & $<0.001$ \\
\hline LVI & & & $<0.001$ \\
\hline No & $236(65.7 \%)$ & $323(88.7 \%)$ & \\
\hline Yes & $123(34.3 \%)$ & $41(11.3 \%)$ & \\
\hline pT stage & & & $<0.001$ \\
\hline I, II & $102(28.4 \%)$ & $159(43.7 \%)$ & \\
\hline III, IV & $257(71.6 \%)$ & $205(56.3 \%)$ & \\
\hline Cell differentiation & & & 0.257 \\
\hline WD & $41(11.4 \%)$ & $55(15.1 \%)$ & \\
\hline MD & $304(84.7 \%)$ & $299(82.1 \%)$ & \\
\hline PD & $14(3.9 \%)$ & $10(2.7 \%)$ & \\
\hline pCR & & & 0.001 \\
\hline No & $359(100 \%)$ & $353(97 \%)$ & \\
\hline Yes & $0(0 \%)$ & $11(3 \%)$ & \\
\hline CRM & $10(2.8 \%)$ & $10(2.7 \%)$ & \\
\hline$(-)$ & & $354(97.3 \%)$ & \\
\hline (+) & & & \\
\hline CRT: C7. & & \\
\hline
\end{tabular}

CRT: Chemoradiotherapy, Pt Stage: Pathologic Tumor Depth Stage, LN: Lymph Node, LVI: Lymphovascular Invasion, Pcr: Pathologic Complete Response, CRM: Circumferential Resection Margin, WD: Well Differentiated, MD: Moderately Differentiated, PD: Poorly Differentiated.

Table 2: Pathology parameters for no CRT and CRT groups.

Final pT stage after CRT was converted to stage 0,1 or 2 in 159 patients of the CRT group (43.7\%) compared to 102 patients $(28.4 \%)$ in the no CRT group $(\mathrm{p}<0.001)$. Pathologic complete response $(\mathrm{pCR})$ was shown in 11 patients (3\%) in the CRT group. Threatened CRM 
Citation: Han YD, Al Bandar MH, Cho MS, Hur H, Min BS, et al. (2016) Impact of Preoperative Chemo-radiation Therapy on Systemic Failure in Locally Advanced Rectal Cancer. J Integr Oncol 5: 180. doi:10.4172/2329-6771.1000180

Page 4 of 8

was documented in 10 patients in each group ( $2.8 \%$ vs. $2.7 \%$; $=0.975$ ). Clinical histopathology results are summarized in Table 2.

\section{Recurrence according to pathologic stage}

Overall local recurrent rate is decreased in CRT arm due to the effect of CRT, $2 \%$ compared to $3 \%$ in no CRT, respectively. Systemic metastasis rate, however, found equal in both study arms, $22.5 \%$ vs. $23 \%$, respectively. The highest rate of local recurrence was evident in pathologic stage 3 in CRT group, rated at $8.6 \%$ vs. $6.1 \%$, in no CRT and CRT group respectively. Systemic recurrent recorded almost similar in both arms in different pathological stage. The CRT group showed a significant rate of systemic failure in p stage $0-1(3.6 \%$ vs. $0 \%, \mathrm{p}<0.005)$ that explained by the initial presentation of high risk features in CRT arm that have been down staged to be equivalent to no CRT.

\begin{tabular}{|c|c|c|c|c|}
\hline $\begin{array}{l}\text { Pathologic } \\
\text { Stage }\end{array}$ & $\begin{array}{l}\text { Recurrence } \\
\text { pattern }\end{array}$ & $\begin{array}{ll}\begin{array}{l}\text { No } \\
(n=359)\end{array} & \text { CRT }\end{array}$ & CRT $(n=364)$ & $P$ value \\
\hline \multirow[t]{3}{*}{ I } & Local & $1(0.3 \%)$ & 0 & $<0.005$ \\
\hline & Local + Systemic & $1(0.3 \%)$ & $4(1.1 \%)$ & \\
\hline & Systemic & 0 & $13(3.6 \%)$ & \\
\hline \multirow[t]{3}{*}{ II } & Local & $4(1.1 \%)$ & $5(1.3 \%)$ & 0.609 \\
\hline & Local + Systemic & $2(0.5 \%)$ & $6(1.64 \%)$ & \\
\hline & Systemic & $14(3.9 \%)$ & $18(4.9 \%)$ & \\
\hline \multirow[t]{3}{*}{ III } & Local & $6(1.7 \%)$ & $3(0.89 \%)$ & 0.602 \\
\hline & Local + Systemic & $16(4.4 \%)$ & $15(4.1 \%)$ & \\
\hline & Systemic & $48(13.4 \%)$ & $31(8.5 \%)$ & \\
\hline \multirow[t]{2}{*}{ Total } & & Local 3\%, & Local (2.1\%) & $>0.005$ \\
\hline & & $\begin{array}{l}\text { Systemic } \\
22.5 \%\end{array}$ & Systemic $(23 \%)$ & \\
\hline
\end{tabular}

CRT: Chemoradiotherapy, p stage: Pathologic Stage.

Table 3: Rates of local and systemic recurrence according to pathologic stage.

However, has not reached efficient systemic control. Overall recurrence was not significantly different between the groups regardless of the poor prognostic features in the CRT group. Local and systemic recurrence rates are illustrated in Table 3.

\section{Adjuvant chemotherapy}

Adjuvant treatment was usually started 6-8 weeks after surgery if the postoperative course was uneventful and without complications. The majority of our patients received 5-fluorouracil-based chemotherapy (77.1\% for no CRT group vs. $78.4 \%$ for CRT group), Xeloda (oxaliplatin and capecitabine)-based chemotherapy (10.7\% vs. $11 \%)$, or FOLFOX or FOLFIRI ( $2.7 \%$ vs. $2.4 \%$ ); other chemotherapy agents were also used in a minority of our patients (5\% vs. $1.2 \%)$. Patient compliance and completion of treatment were successfully achieved in $97.3 \%$ vs. $97.6 \%$ of patients, respectively $(\mathrm{p}=0.072)$. Adjuvant chemotherapy was recommended less often in the no CRT group than the CRT group $(72.7 \%$ vs. $89.8 \%, \mathrm{p}<0.001)$; this difference was particularly evident for patients with p stage $0-1$ (1.5\% vs. $88.5 \%$, $\mathrm{p}<0.001$ ), whereas adjuvant chemotherapy was administered equally between the groups in those with p stage 2 or $3(85.4 \%$ vs. $89.9 \%$, $\mathrm{p}=0.375$ and $90.6 \%$ vs. $92.1 \%, \mathrm{p}=0.273$ respectively). The chosen chemotherapy agents are listed in Table 4.

\begin{tabular}{|l|l|l|l|}
\hline Adj CTx & No CRT (n=359) & CRT (n=364) & p value \\
\hline No & $98(27.3 \%)$ & $37(10.2 \%)$ & $<0.001$ \\
\hline Yes & $261(72.7 \%)$ & $327(89.8 \%)$ & \\
\hline Adj CTx Regimen & & & 0.072 \\
\hline 5FU-based & $202(77.1 \%)$ & $257(78.4 \%)$ & \\
\hline Xeloda-based & $28(10.7 \%)$ & $36(11 \%)$ & \\
\hline FOLFOX or FOLFIRI & $12(4.6 \%)$ & $23(7 \%)$ & \\
\hline Discontinued & $7(2.7 \%)$ & $8(2.4 \%)$ & \\
\hline Others & $13(5 \%)$ & $4(1.2 \%)$ & \\
\hline CRT: Chem & & & \\
\hline
\end{tabular}

CRT: Chemoradiotherapy, Adj Ctx: Adjuvant Chemotherapy, 5 FU: 5Fluorouracil, Xeloda: Oxaliplatin and Capecitabine, FOLFOX: Oxaliplatin in Combination With 5-Fluorouracil and Leucovorin, FOLFIRI: Irinotecan in Combination with 5-Fluorouracil and Leucovorin.

Table 4: Adjuvant Chemotherapy Regimens.

\begin{tabular}{|l|l|l|l|l|l|l|}
\hline & Pathologic Stage & CRT & $95 \%$ Cl & No CRT & $95 \%$ Cl & p value \\
\hline \multirow{2}{*}{$\begin{array}{l}\text { Disease-free } \\
\text { Survival }\end{array}$} & 0, I & $90.50 \pm 3.16$ & $84.31-96.69$ & $106.42 \pm 2.15$ & $102.21-110.62$ & 0.034 \\
\cline { 2 - 7 } & II & $80.56 \pm 4.02$ & $72.68-88.44$ & $95.7 \pm 4.11$ & $87.65-103.75$ & 0.016 \\
\cline { 2 - 7 } & III & $61.31 \pm 4.3$ & $52.89-69.73$ & $76.26 \pm 3.62$ & $69.17-83.36$ & 0.14 \\
\hline \multirow{2}{*}{ Overall Survival } & 0, I & $101.88 \pm 1.49$ & $98.96-104.79$ & $107.2 \pm 1.99$ & $103.3-111.1$ & 0.148 \\
\cline { 2 - 7 } & II & $87.91 \pm 5.61$ & $76.90-98.91$ & $104 \pm 3.17$ & $97.79-110.21$ & 0.165 \\
\cline { 2 - 7 } & III & $85.77 \pm 3.89$ & $78.15-93.4$ & $93.66 \pm 2.91$ & $87.95-99.36$ & 0.802 \\
\hline
\end{tabular}

95\% Cl: 95\% confidence interval, CRT: Chemoradiotherapy, p stage: Pathologic Stage.

Table 5: Survival Analysis. 


\section{Disease-free survival and overall survival analysis}

Disease free survival (DFS) for $\mathrm{p}$ stage 0 -1 was significantly different between groups due to the higher overall recurrence rate in the CRT group. DFS in p stage $0-1$ was $106.42 \pm 2.15,95 \%$ CI $102.21-110.62$ for no CRT vs. $90.50 \pm 3.16,95 \%$ CI 84.31-96.69 for CRT ( $\mathrm{p}=0.034)$. When it comes to $\mathrm{p}$ stage 2 also showed a significant different in DFS between the two study groups $(95.7 \pm 4.11,95 \%$ CI $87.65-103.75$ vs. $80.56 \pm 4.02$ $95 \%$ CI 72.68-88.44, $\mathrm{p}=0.016)$. However, DFS was not significantly different for $\mathrm{p}$ stage $3(76.26 \pm 3.62,95 \%$ CI $69.17-83.36$ vs. $61.31 \pm 4.3$, 95\% CI 52.89-69.73, p=0.14). DFS analysis is presented and summarized in Table 5 .

Overall survival was not significantly different between both groups. The median OS for no CRT and CRT groups was $107.2 \pm 1.99,95 \%$ CI 103.3-111.1 vs. $101.88 \pm 1.49,95 \%$ CI 98.96-104.79 $(\mathrm{p}=0.148)$, respectively, for p stage $0-1 ; 104 \pm 3.17,95 \%$ CI $97.79-110.21$ vs. 87.91 $\pm 5.61,95 \%$ CI 76.90-98.91 ( $\mathrm{p}=0.165)$ for $\mathrm{p}$ stage 2 ; and $93.66 \pm 2.91$ 95\% CI 87.95-99.36 vs. $85.77 \pm 3.89,95 \%$ CI 78.15-93.4 ( $\mathrm{p}=0.802)$ for $\mathrm{p}$ stage 3. Overall survival is summarized in Table 5 and Figure 1.



Figure 1: Kaplan-Meier estimates disease-free survival and overall survival.

\section{Discussion}

This is a single-center experience at which all patients were carefully monitored, and the treatment protocols for the two groups were strictly followed with precise documentation in our database with no violation. The use of cutting edge technology by expert surgeons with a high level of experience in the colorectal field greatly reduced the possibility of surgical factors being confounders of our study outcome. This enabled us to clearly demonstrate the efficacy of neoadjuvant chemotherapy in locally advanced low rectal cancer.

Observations in our study of 723 patients with mid- and low advanced rectal cancer revealed the efficacy of CRT with fluoropyrimidine-based concomitant chemotherapy in controlling the tumor locally, which was especially prominent in pathological stage 0 -1. Interestingly, CRT has contributed to down-staged rate of local recurrent in high-risk group to a level equal to that of the low risk group. Systemic recurrent was not managed by CRT and have been stated equally in both groups in pathological stage II-III, although higher rate have shown in pathological stage I in CRT group. Higher systemic rate in p stage I in CRT group owing to existing high risk feature in MRI before planned surgery. Failure of systemic control in these patients group is the major concern. Indeed, systemic metastasis was accounted for the highest tumor dissemination in our patients reaching $23 \%$ comparing to only $3 \%$ recorded for local recurrent. Therefore, deviation of treatment strategy to focus on systemic spread rather than local recurrent, particularly if locally advanced with suspected metastasis lymph node (our patient sample). Then we raise questions about the true influence of CRT on tumor control and whats is the best alternative option to approach those types of patients.

\section{Suggested theory in chemo-radiotherapy failure}

We propose four responsible factors contributed of systemic failure in the CRT group: first, the time interval between CRT and surgery contributed to delayed surgery and increased the risk of systemic tumor propagation; second, the chemotherapy component of the CRT regimen is a sensitizing agent to radiotherapy that improves local control without systemic influence; third, rectal tumors in CRT group were more locally advanced with high risk features without adding any further chemotherapy agents to overcome the tumor burden during the period of CRT administration . Fourth, majority of primary tumors in the CRT group located at the lower rectum compared to those in the no CRT group (150(41.2\%) vs. 66(18.4\%), $\mathrm{p}<0.001$, respectively). These four key factors are thought to be responsible to set an appropriate management plan. To overcome these obstacles, we need to improve CRT quality by utilizing induction or concomitant chemotherapy agents, as well as to consider the possibility of shortening the time interval between CRT and surgery.

\section{Up front radiotherapy or chemo-radiotherapy agent}

Whether the time to surgery should be shortened or extended has been a subject of debate for decades. In order to achieve early intervention, several studies have considered short radiotherapy treatment followed by early surgery versus CRT and delayed surgery. Numerous studies have shown favor for CRT over a radiotherapy course only. In addition, administration of radiotherapy only was a topic of interest at which we could shorten the time to surgery and then earlier induction of adjuvant chemotherapy would be anticipated. However, a systematic review of six randomized controlled trials regarding the efficacy of CRT in non-metastatic locally advanced rectal cancer showed a significant reduction in LR for CRT compared with preoperative radiation-only [17]. Furthermore, another study predicted successful intervention with short radiotherapy administration and delayed surgery [18].

\section{Chemo-radiotherapy therapy administration, before or after assigned surgery}

A German trial [4] compared preoperative CRT with postoperative CRT in 404 patients with advanced rectal cancer (T3, T4, N+) and showed similar OS at 10 years of $59.6 \%$ in the preoperative arm and $59.9 \%$ in the postoperative arm $(\mathrm{p}=0.85)$. The 10 -year cumulative incidence of local relapse was $7.1 \%$ and $10.1 \%$ in the pre- and postoperative arms, respectively $(\mathrm{p}=0.048)$. No significant differences were detected for 10-year cumulative incidence of distant metastases (29.8\% and 29.6\%; $\mathrm{p}=0.9$ ). Eligibility criteria for the German trial were similar to those of our study, including positive lymph nodes, but the treatment strategy was different. As the German trial did not demonstrate any beneficial effect on OS by CRT either before or after surgery, a different approach to achieve systemic metastasis control in 
advanced rectal cancer is required, particularly if the tumor is located in the lower rectum, as demonstrated in our study.

\section{Debates in surgery first or chemo-radiotherapy up front}

In order to shorten the time to surgery, postponing the CRT regimen until after surgery has been proposed. In the usual manner, the CRT course takes 5 weeks, followed by surgery after a 2 -week recovery time, and then CRT will be administered after an additional 6-8 weeks. Although this long time period before CRT intervention is expected to contribute to distal tumor progression, preoperative CRT yields favorable outcomes. These debates will be explained in the next section.

In contrast to a shortened time interval, a prolonged time interval allows tumor regression before surgery, which might account for improved recurrence rate and overall survival. The Stockholm III trial [18] showed pathologic complete response (pCR) in $0.8 \%$ of patients after short-course radiotherapy (SCRT) followed by surgery within 1 week; however, a $12.5 \%$ pCR was noted when surgery was performed after 4-8 weeks. The Stockholm study also reported that the risk of tumor progression during the "waiting time" is low even if chemotherapy is not administered in this period. Although CRT alone improved local control and cancer-specific survival in a previous study, older patients might be too fragile to undergo treatment with CRT. Recent studies conducted in Europe showed favorable outcomes by delivering radiotherapy of 5 Gy $\times 5$ with delayed surgery [19-21]. Furthermore, for locally advanced tumors, downstaging is required to facilitate surgical resection with negative resection margins. A Polish trial that compared SCRT versus long-course CRT found more downstaging after CRT (16\%) compared with SCRT (1\%) [19]. This difference in downstaging did not result in differences in local recurrence control, disease-free survival, or OS between the treatment groups; however, other studies have reported a $45 \%$ downstaging effect of CRT compared with $28 \%$ in SCRT [22,23]. Based on this background, investigation into the rationale for SCRT for locally advanced rectal cancer is an interesting ongoing project.

\section{Systemic failure on locally advanced rectal cancer}

Considering the influence of CRT on local control but not systemic outbreak, our results are concordant with those of the EORTC Radiotherapy Group Trial 22921, in which the 5-year cumulative incidence of local recurrence was $17.1 \%$ in the radiotherapy group but was reduced to $7-9.6 \%$ when chemotherapy was administered either pre- or postoperatively $[24,25]$. However, there was no difference in OS or systemic control between four treatment groups of radiotherapy only, CRT only, radiotherapy + adjuvant, or CRT + adjuvant. Nevertheless, we correlated our results with pathologic stage and poor prognostic features that had been reported in no CRT group. The FFCD 9203 Trial [25], a randomized trial comparing preoperative radiotherapy with CRT in 733 patients, reported improvement of local recurrence but not OS in clinical stage T3 or T4; this is consistent with our results. However, unknown lymph node status or no lymph node predicted accounted for more than $50 \%$ of the sample in the previous study, whereas we deemed lymph node positivity by MRI a criterion for admission eligibility. A meta-analysis of seven randomized control trials and 10 retrospective studies including more than 5,000 patients diagnosed with rectal cancer who received CRT, surgery, and then adjuvant chemotherapy showed improved 5-year OS and tumor downstaging [26].

\section{Independent risk factors of local or systemic failure}

Numerous other subjective measures have been suggested to be independent risk factors of local or systemic failure. We highlighted two variables, pCR and CRM status, in our study to rule out their confounding effects. We maintained high surgical quality with highly skillful surgeons throughout the study period, resulting in only 10 patients with positive CRM in each group $(2.8 \%$ vs. $2.7 \%, \mathrm{p}=0.972)$. However, only 11 (3\%) were classified as pCR after CRT regimens based on imaging studies and final histopathology reports. Concerning the achievement of pCR, decision making via MRI staging and implementation of CRT for early stage tumors may generate promising results. In a pooled analysis of 3,105 patients in 17 prospective randomized clinical trials, $16 \%$ of the patients achieved pCR in a median follow-up of 48 months. pCR was the only predictor of disease-free survival with a hazard ratio of 0.54 , but was only achieved in 11 patients (3\%) in our study [27]. Interestingly, a single-center retrospective cohort study that compared surgery alone and CRT in patients with CRM-positive and N2 status in terms of CRM involvement and local recurrence rates revealed a significant difference in local recurrence between CRT and surgery-alone groups (6.5\% vs. $0 \%, \mathrm{p}=0.04$, respectively) and showed that CRM involvement was the only predictive factor of local recurrence [28].

\section{Strength and limitation of our study}

Strengths of our study include the assessment of CRT effect in locally advanced mid and low rectal cancers; classified to low and high group. All participating surgeon are a well-qualified surgeon performing high-quality surgery based on safe oncological principles. Data gathered from a well-known high volume tertiary center. Although this study carried out in retrospective manner, computerized based system and crucial patient follow up, help us to minimize missing data. In addition, we correlated tumor recurrent to the initial site of the tumor, which might influenced our results. We reported lower level of rectal tumor in CRT group than no CRT group, particularly in $\mathrm{p}$ stage 0 - 1 . In our data analysis, we did not focus on chemotherapy-related toxicity or surgery complications in the two study arms. A further weakness of our study was that the use of transrectal endoscopy for preoperative staging of patients was not uniform among the surgeons. There could have been selection bias in the CRT group as they were deemed to have highly advanced disease with poor prognostic features. However, we overcame this obstacle by involving only $\mathrm{N}$-positive cases that were equivalent to stage III rectal cancer with adverse clinical features at presentation.

This study contributes to the growing evidence suggesting that a highly selective approach to the use of CRT is more appropriate than widespread application given concerns regarding toxicity in additional to a major concern about CRT regimen modification in a high-risk group of rectal cancer patients to reduce tumor spread and improve overall survival, which is a topic of serious debate among trials [29]. The rationale for CRT opens a window for testing combinations of numerous concepts in advanced rectal cancer. Conflicting results and ongoing debates remain, together with the lack of a proper standard protocol for these challenging cases.

\section{Current ongoing trials}

At the present time, new initiatives such as the RAPIDO (Radiotherapy and Preoperative Induction therapy followed by Dedicated Operation) trial, which compares CRT with SC-PRT 
followed by chemotherapy and delayed surgery, are accruing patients. Interestingly, a phase II trial was undertaken to evaluate the safety and efficacy of perioperative XELOX without radiotherapy for patients with high-risk LARC. Patients received four cycles of XELOX before and after surgery [30]. Whether an induction chemotherapy agent should be commenced in a neoadjuvant setting, particularly with the development of new biological agents, is the subject of a UK phase III trial (Bevacizumab and Combination Chemotherapy in Rectal Cancer Until Surgery, BACCHUS; NCT01650428).

\section{Conclusion}

Distant metastasis continues to be a major obstacle in the treatment of patients with advanced rectal cancer. Timing of surgery and adequate CRT course has to be investigated in order to reduce the risk of distal metastasis. Our data showed an effect of CRT on local control in p stage $0-1$ but raised questions about the appropriate use of CRT in a high-risk group. Therefore, from our study we could identify patients at risk for distant metastasis that should be considered to optimize the course of pretreatment strategy to reduce distant metastasis in advanced rectal cancer. CRT modification is under serious investigation to address current obstacles in the treatment of advanced rectal cancer.

\section{Acknowledgement and Author Contribution}

This study was based entirely on the Authors' work, with no involvement of any third party in data analysis or writing the article. $\mathrm{E}$ world editing was attributed in English editing. Mahdi Hussain AlBandar: Study design, writing the manuscript and interpreting data results; Yoon Dae Han: statistical analysis, interpreting data results manuscript editing;. Min Soo Cho, Hyuk Hur, Byung Soh Min and Kang Young Lee, were contributed in study design and data critics the data and manuscript; Nam Kyu Kim: charged in data gathering, interpreting the data and manuscript critics.

\section{Conflict of Interest Statement}

The Authors do not have any potential or actual personal, political, or financial interest in the material, information, or techniques described in the paper.

\section{References}

1. Siegel R, Naishadham D, Jemal A (2013) Cancer statistics, 2013, CA. Cancer J Clin 63: 11-30.

2. Heald RJ, Husband EM, Ryall RD (1982) The mesorectum in rectal cancer surgery-the clue to pelvic recurrence? Br J Surg 69: 613-616.

3. Compton CC (2003) Colorectal carcinoma: diagnostic, prognostic, and molecular features. Modern Pathol 16: 376-388.

4. Sauer R, Liersch T, Merkel S, Fietkau R, Hohenberger W, et al. (2012) Preoperative versus postoperative chemoradiotherapy for locally advanced rectal cancer: results of the German CAO/ARO/AIO-94 randomized phase III trial after a median follow-up of 11 years. J Clin Oncol 30: 1926-1933.

5. Peeters KC, Marijnen CA, Nagtegaal ID, Kranenbarg EK, Putter H, et al. (2007) The TME trial after a median follow-up of 6 years: increased local control but no survival benefit in irradiated patients with resectable rectal carcinoma. Ann Surg 246: 693-701.

6. Havenga K, Enker WE, Norstein J, Moriya Y, Heald RJ, et al. (1999) Improved survival and local control after total mesorectal excision or D3 lymphadenectomy in the treatment of primary rectal cancer: an international analysis of 1411 patients. Eur J Surg Oncol 25: 368-374.
7. Garajova I, Di Girolamo S, de Rosa F, Corbelli J, Agostini V, et al. (2011) Neoadjuvant treatment in rectal cancer: actual status. Chem Res Pract 12: 839-742.

8. Gerard A, Pector JC, Van Houtte P, Bleiberg H (1993) Adjuvant treatments in resectable cancer of the rectum: preoperative radiotherapy, postoperative radiation- and chemotherapy or nothing?. Annals De Chirurgie 47: 294-301.

9. Sebag-Montefiore D, Stephens RJ, Steele R, Monson J, Grieve R, et al. (2009) Preoperative radiotherapy versus selective postoperative chemoradiotherapy in patients with rectal cancer [MRC CR07 and NCIC-CTG C016]: a multicentre, randomised trial. Lancet 373: 811-820.

10. Rodel C, Liersch T, Becker H, Fietkau R, Hohenberger W, et al. (2012) Preoperative chemoradiotherapy and postoperative chemotherapy with fluorouracil and oxaliplatin versus fluorouracil alone in locally advanced rectal cancer: initial results of the German CAO/ARO/AIO-04 randomised phase 3 trial. Lancet Oncol 13: 679-687.

11. Nakamura T, Yamashita K, Sato T, Ema A, Naito M, et al. (2014) Neoadjuvant chemoradiation therapy using concurrent S-1 and irinotecan in rectal cancer: impact on long-term clinical outcomes and prognostic factors. Int J Rad Oncol, Biol, Phy 89: 547-555.

12. Chua YJ, Barbachano Y, Cunningham D, Oates JR, Brown G, et al. (2010) Neoadjuvant capecitabine and oxaliplatin before chemoradiotherapy and total mesorectal excision in MRI-defined poor-risk rectal cancer: a phase 2 trial. Lancet Oncol 11: 241-248.

13. Marechal R, Vos B, Polus M, Delaunoit T, Peeters M, et al. (2012) Short course chemotherapy followed by concomitant chemoradiotherapy and surgery in locally advanced rectal cancer: a randomized multicentric phase II study. Annals Oncol 23: 1525-1530.

14. Kamiya T, Uehara K, Nakayama G, Ishigure K, Kobayashi S, et al. (2016) Early results of multicenter phase II trial of perioperative oxaliplatin and capecitabine without radiotherapy for high-risk rectal cancer: CORONA I study. Eur J Surg Oncol 6: 1-4.

15. Peeters KC, van de Velde CJ, Leer JW, Martijn H, Junggeburt JM, et al. (2005) Late side effects of short-course preoperative radiotherapy combined with total mesorectal excision for rectal cancer: increased bowel dysfunction in irradiated patients--a Dutch colorectal cancer group study. J Clin Oncol 23: 6199-6206.

16. Edge SB, Compton CC (2010) The American Joint Committee on Cancer: the 7th edition of the AJCC cancer staging manual and the future of TNM. Ann Surg Oncol 17: 1471-1474.

17. McCarthy K, Pearson K, Fulton R, Hewitt J (2012) Pre-operative chemoradiation for non-metastatic locally advanced rectal cancer. Cochrane Database Syst Rev 12: Cd008368.

18. Pettersson D, Cedermark B, Holm T, Radu C, Pahlman L, et al. (2010) Interim analysis of the Stockholm III trial of preoperative radiotherapy regimens for rectal cancer. Br J Surg 97: 580-587.

19. Braendengen M, Tveit KM, Berglund A, Birkemeyer E, Frykholm G, et al. (2008) Randomized phase III study comparing preoperative radiotherapy with chemoradiotherapy in nonresectable rectal cancer. J Clin Oncol 26: 3687-3694.

20. Radu C, Berglund A, Pahlman L, Glimelius (2008) Short-course preoperative radiotherapy with delayed surgery in rectal cancer - a retrospective study. Radiother Oncol 87: 343-349.

21. Pettersson D, Holm T, Iversen H, Blomqvist L, Glimelius B, et al. (2012) Preoperative short-course radiotherapy with delayed surgery in primary rectal cancer. Br J Surg 99: 577-583.

22. Bujko K, Nowacki MP, Nasierowska-Guttmejer A, Michalski W, Bebenek $\mathrm{M}$, et al. (2006) Long-term results of a randomized trial comparing preoperative short-course radiotherapy with preoperative conventionally fractionated chemoradiation for rectal cancer. Br J Surg 93: 1215-1223.

23. Ngan SY, Burmeister B, Fisher RJ, Solomon M, Goldstein D, et al. (2012) Randomized trial of short-course radiotherapy versus long-course chemoradiation comparing rates of local recurrence in patients with T3 rectal cancer: Trans-Tasman Radiation Oncology Group trial 01-04. J Clin Oncol 30: 3827-3833. 
Citation: Han YD, Al Bandar MH, Cho MS, Hur H, Min BS, et al. (2016) Impact of Preoperative Chemo-radiation Therapy on Systemic Failure in Locally Advanced Rectal Cancer. J Integr Oncol 5: 180. doi:10.4172/2329-6771.1000180

Page 8 of 8

24. Bosset JF, Collette L, Calais G, Mineur L, Maingon P, et al. (2006) Chemotherapy with preoperative radiotherapy in rectal cancer. $\mathrm{N}$ Engl Med 355: 1114-1123.

25. Gerard JP, Conroy T, Bonnetain F, Bouche O, Chapet O, et al. (2006) Preoperative radiotherapy with or without concurrent fluorouracil and leucovorin in T3-4 rectal cancers: results of FFCD 9203. J Clin Oncol 24 4620-4625.

26. Petrelli F, Coinu A, Lonati V, Barni S (2015) A systematic review and meta-analysis of adjuvant chemotherapy after neoadjuvant treatment and surgery for rectal cancer. Int J Colorectal Dis 30: 447-457.

27. Maas M, Nelemans PJ, Valentini V, Das P, Rodel C, et al. (2010) Longterm outcome in patients with a pathological complete response after chemoradiation for rectal cancer: a pooled analysis of individual patient data. Lancet Oncol 11: 835-844.

28. Williamson JS, Jones HG, Davies M, Evans MD, Hatcher O, et al. (2014) Outcomes in locally advanced rectal cancer with highly selective preoperative chemoradiotherapy. Br J Surg 101: 1290-1298.

29. Swellengrebel HA, Marijnen CA, Verwaal VJ, Vincent A, Heuff G, et al. (2011) Toxicity and complications of preoperative chemoradiotherapy for locally advanced rectal cancer. Br J Surg 98: 418-426.

30. Nilsson PJ, van Etten B, Hospers GA, Pahlman L, van de Velde CJ, et al. (2013) Short-course radiotherapy followed by neo-adjuvant chemotherapy in locally advanced rectal cancer-the RAPIDO trial. BMC cancer 13: 279. 